\title{
Role of Primary and Secondary Surfactant Layers on the Thermal Conductivity of Lauric Acid Coated Magnetite Nanofluids
}

\author{
R. Lenin ${ }^{1,2}$ and P. A. Joy ${ }^{1,2, *}$ \\ ${ }^{1}$ Physical and Materials Chemistry Division, CSIR- National Chemical Laboratory \\ Pune 411008, India \\ ${ }^{2}$ Academy of Scientific and Innovative Research (AcSIR), CSIR- National Chemical \\ Laboratory, Pune 411008, India
}

Powder X-Ray Diffraction

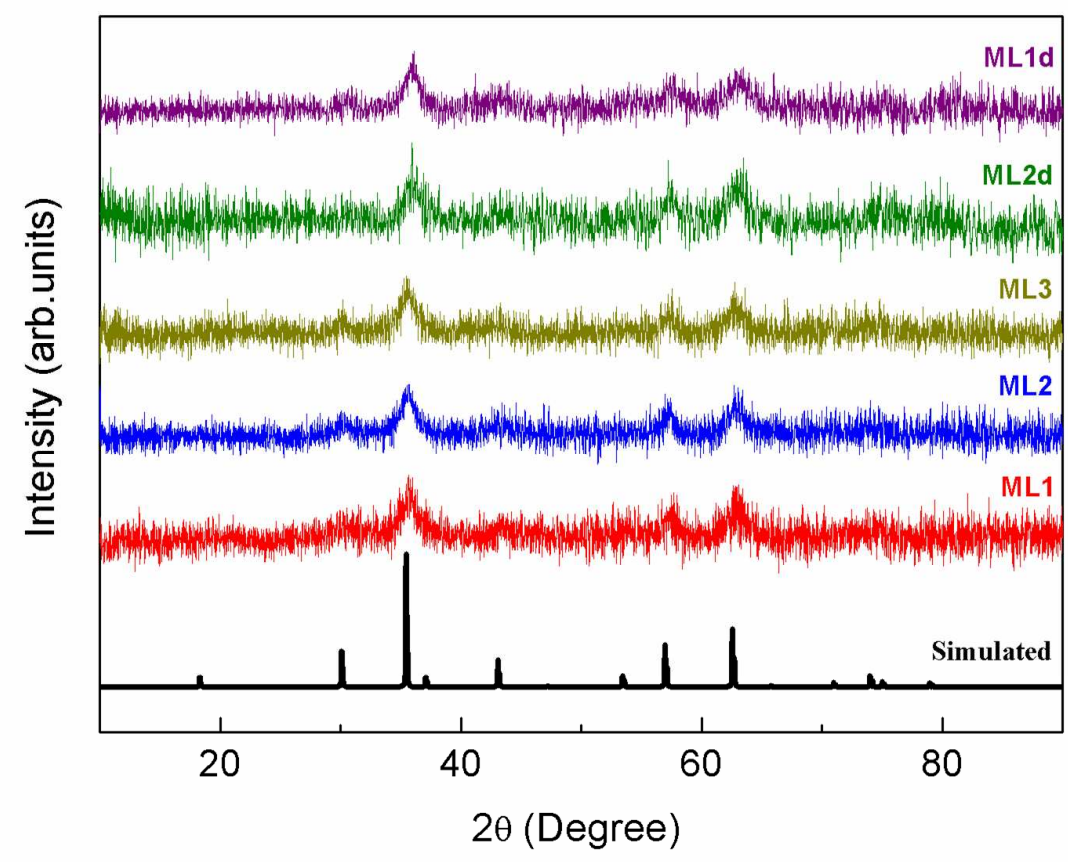

Figure S1. Powder X-ray diffraction patterns of the dried lauric acid coated samples, compared with the simulated pattern of $\mathrm{Fe}_{3} \mathrm{O}_{4}$ (black) 


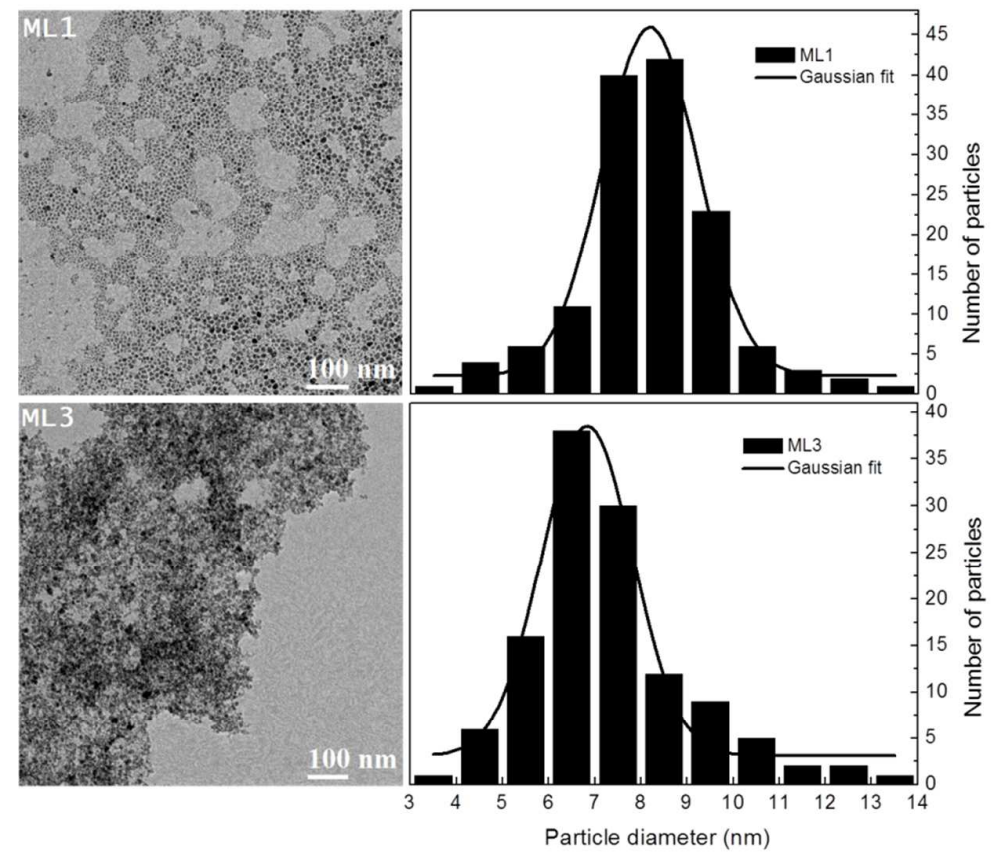

Figure S2. TEM images of the lauric acid coated samples ML1 and ML3 (left) and the corresponding particle size distribution histograms (right). 


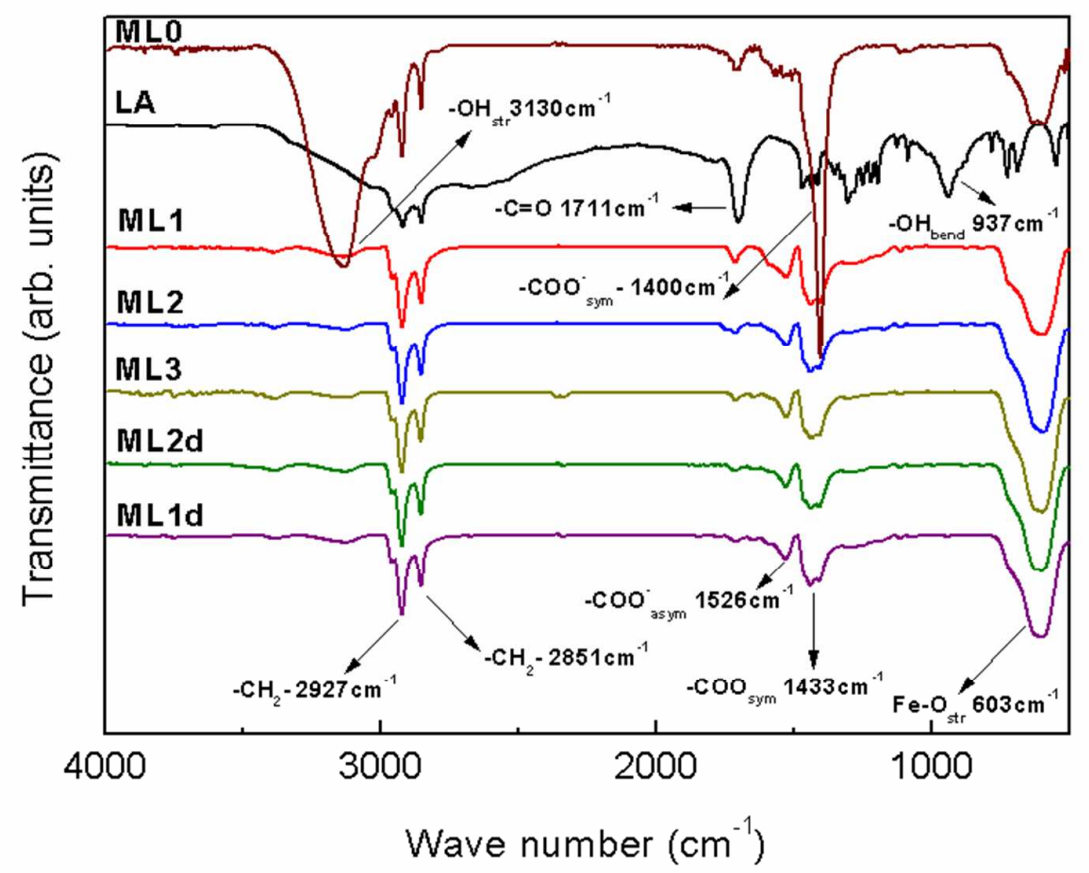

Figure S3. Infrared spectra of neat lauric acid, LA (black), compared with the spectra of different lauric acid coated samples (ML0, ML1, ML2, ML3, ML2d, ML1d).

\section{Magnetic measurements}

Magnetization measurements were done for all the lauric acid coated samples after cooling the samples to $5 \mathrm{~K}$ from room temperature under zero field cooled (ZFC) and field cooled (FC) conditions at 50 Oe. All the data show characteristics of superparamagnetic particles, where the temperature at which a maximum is observed in the ZFC magnetization curve is the superparamagnetic blocking temperature $\left(\mathrm{T}_{\mathrm{B}}\right)$.

The shape of the FC magnetization curve gives information on the interparticle interactions. Continuously increasing $\mathrm{FC}$ magnetization below $\mathrm{T}_{\mathrm{B}}$ is an indication for the absence of interparticle interactions whereas a flat FC curve suggests strong interactions. The shape of the FC magnetization curve below $\mathrm{T}_{\mathrm{B}}$ is different for the three samples ML1, ML2, and ML3 (Figure S4(b)). For ML1 with larger amount of the secondary surfactant, the FC magnetization curve increases continuously below $T_{B}$ whereas a saturating trend is observed at lower temperatures for ML3. This suggests reduced interparticle interactions in the order 
ML1 < ML2 < ML3 where the continuously decreasing FC magnetization with increasing temperature, below $\mathrm{T}_{\mathrm{B}}$, is an indication for the strength of the interparticle interactions, which gives information on the effectiveness of coating and the separation between the nanoparticles $[1,2]$. Due to the lower amount of the secondary surfactant layer in ML3, the interparticle interactions are relatively larger in this sample.

Similar differences in the ZFC and FC magnetization curves are observed for the samples washed after a delay of 5 minutes. For ML2d and ML1d, the blocking temperatures are obtained as $84 \mathrm{~K}$ and $87 \mathrm{~K}$, respectively (Figure S4(a)) and corresponding changes in the nature of the FC magnetization curves are also observed (Figure S4(c)), suggesting the role of the surfactant layers in determining the interparticle magnetic interactions.
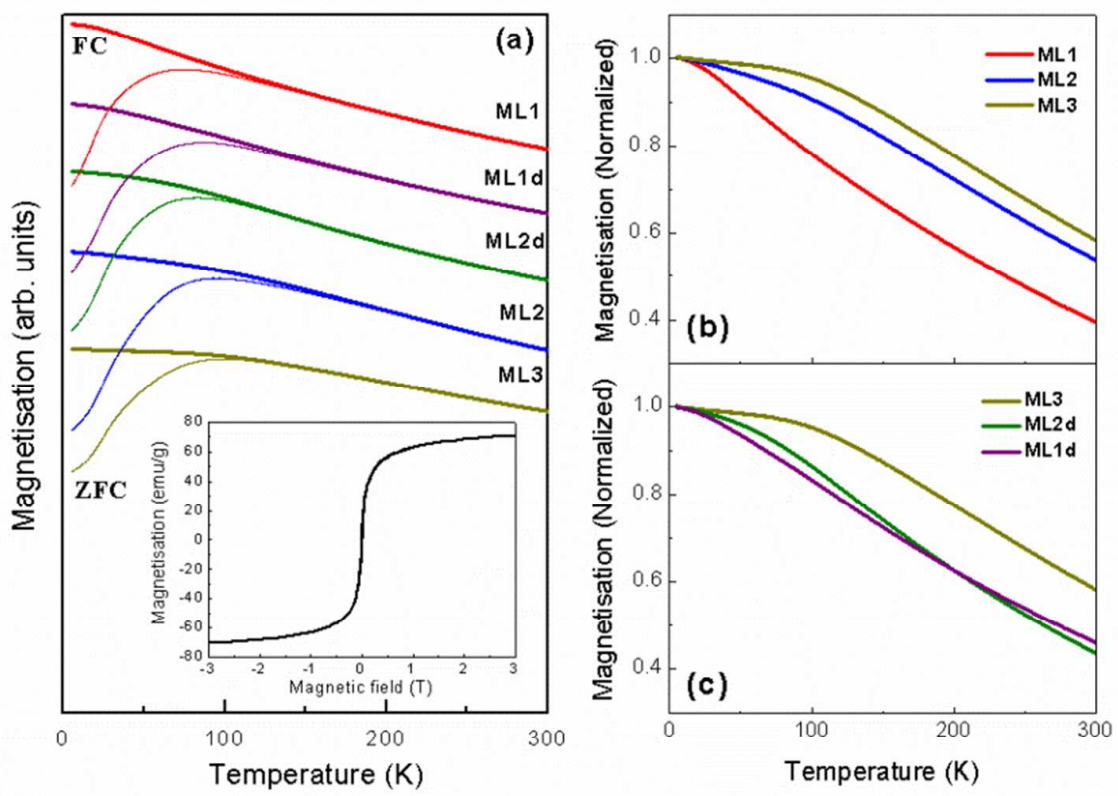

Figure S4. Magnetic measurements on the lauric acid coated powder samples. (a) zero field cooled (ZFC) and field cooled (FC) measurements, inset shows room temperature magnetization measurement as a function of field, (b) normalized field cooled curves of the first set of samples (ML1, ML2, ML3), (c) normalized field cooled curves of the second set of samples (ML3, ML2d, ML1d). 


\section{Freshly prepared samples}
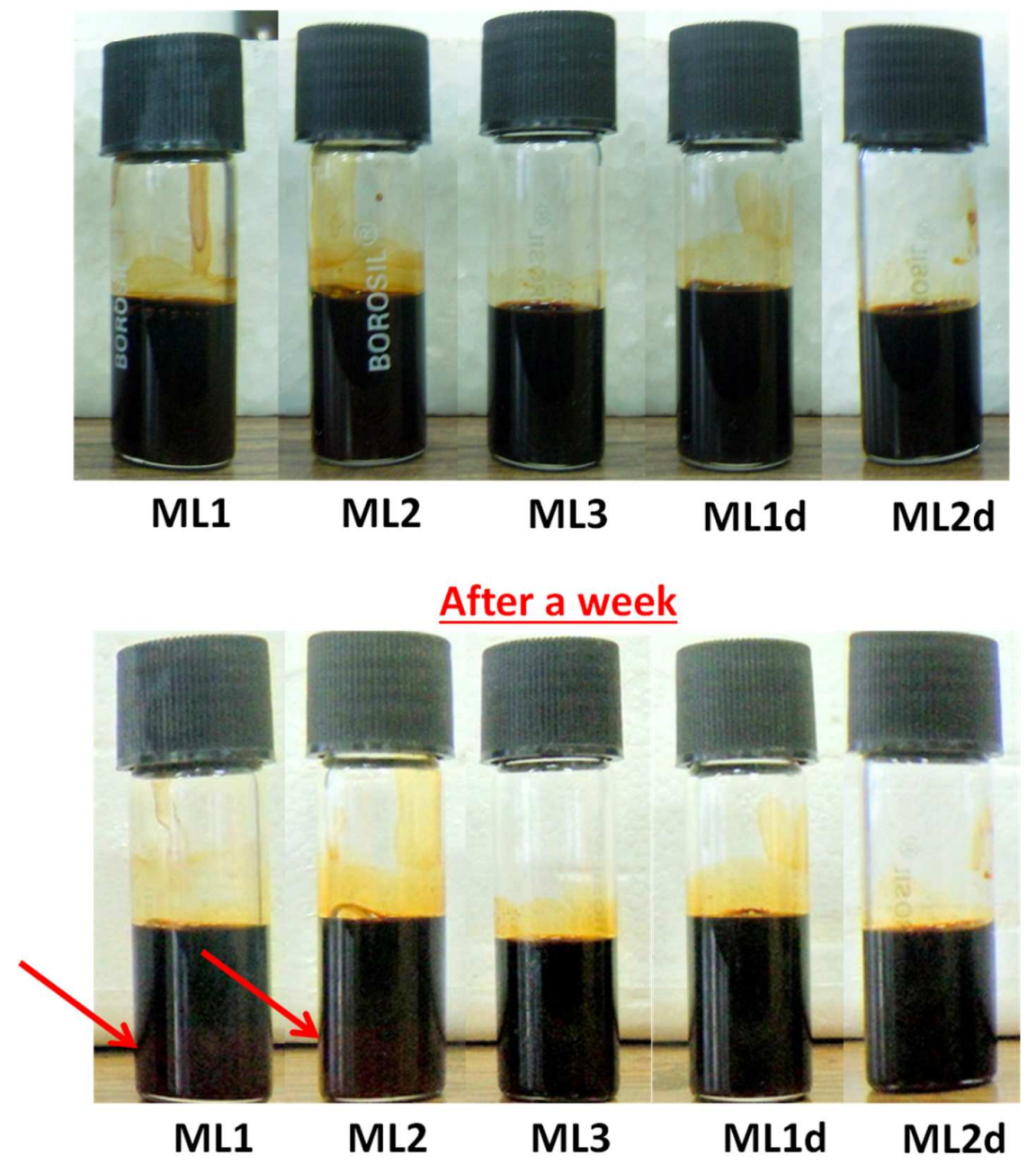

Figure S5. Photographs of the freshly prepared fluids (top) and taken a week after the preparation (bottom) of the fluids. The arrows indicated in the images of the fluids ML1 and ML2 (bottom image) show slight sedimentation of the nanoparticles in the fluid. 
(a)

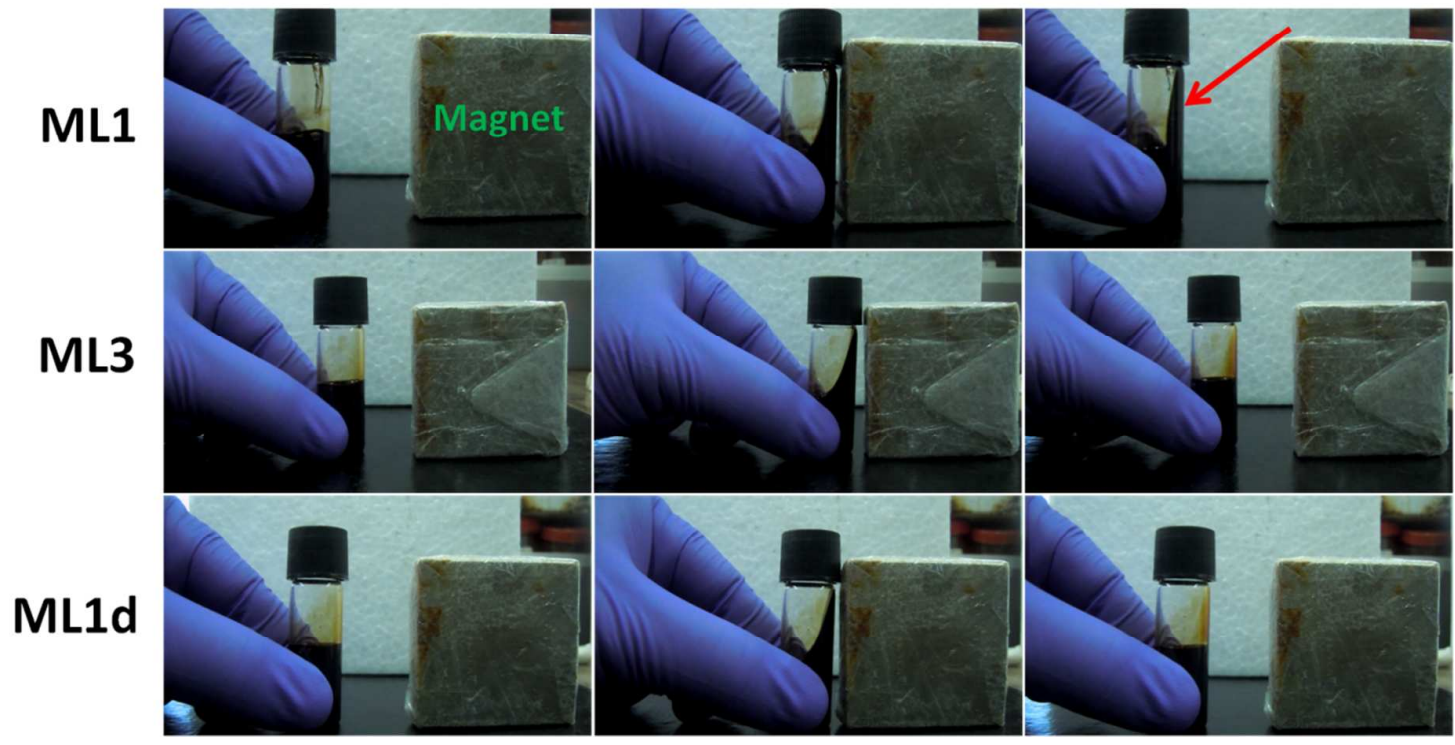

Figure S6. Photographs showing the fluid samples ML1, ML3 and ML1d (a) kept away from a magnet, (b) kept closer to the magnet, and (c) after moved away from the magnet. The arrow in the image of ML1 (c) indicates slightly adhered fluid sample on the container wall.

\section{Models and parameters used for calculation of thermal conductivity}

In the simple Maxwell model for calculation of the thermal conductivity of a nanofluid $\left(k_{e f f}\right)$, one considers only the thermal conductivity of the particle $\left(k_{p}\right)$, thermal conductivity of base fluid $\left(k_{f}\right)$ and the volume percentage $(\phi)$ of the particles.

$$
\frac{k_{e f f}}{k_{f}}=1+\frac{3\left(k_{p} / k_{f}-1\right) \phi}{\left(k_{p} / k_{f}+2\right)-\left(k_{p} / k_{f}-1\right) \phi}
$$

Thermal conductivity of $\mathrm{Fe}_{3} \mathrm{O}_{4}\left(k_{p}\right)=7 \mathrm{Wm}^{-1} \mathrm{~K}^{-1}[3]$

Thermal conductivity of toluene $\left(k_{f}\right)=0.13 \mathrm{Wm}^{-1} \mathrm{~K}^{-1}$ (measured value $@ 25{ }^{\circ} \mathrm{C}$, comparable to the literature reported value) [4]

Interfacial resistance at the interface influences much on the thermal conductivity of composite materials. The interfacial resistance at the interface reduces the effective thermal conductivity of the composite materials [5]. The effective medium approximation including the interfacial resistance $\left(\mathrm{R}_{\mathrm{b}}\right)$ for the spherical particle is given as $[6,7]$,

$$
\frac{k_{e f f}}{k_{h}}=\frac{k_{p}(1+2 \alpha)+2 k_{h}+2 \phi\left[k_{p}(1-\alpha)-k_{h}\right]}{k_{p}(1+2 \alpha)+2 k_{h}-\phi\left[k_{p}(1-\alpha)-k_{h}\right]}
$$


where $k_{h}$ is the thermal conductivity of the host material, $k_{p}$ is the thermal conductivity of the particles, and $\alpha=2 R_{b} k_{h} / d . R_{b}$ is the interfacial thermal resistance and $d$ is the diameter of the particles. In case of nanofluids, thermal conductivity of the host material (base fluid) is much lower than the thermal conductivity of the solid inclusions (nanoparticles), i.e. $k_{h}=k_{f}$ $<<k_{p}$, and the above equation is modified by Prasher et al as [8]

$$
\frac{k_{e f f}}{k_{f}}=\frac{(1+2 \alpha)+2 \phi(1-\alpha)}{(1+2 \alpha)-\phi(1-\alpha)}
$$

In the calculation of the thermal conductivity using the above equation, we have used the interfacial thermal resistance $R_{b}=2.16 \times 10^{-8} \mathrm{~W}^{-1} \mathrm{~m}^{2} \mathrm{~K}$ [1] and the particle diameter as 8.5 nm.

The semi-empirical relation for the Brownian motion induced microconvection of nanoparticles in a fluid is given by [8],

$$
\frac{k_{e f f}}{k_{f}}=\left(1+A \operatorname{Re}^{m} \operatorname{Pr}^{0.333} \phi\right)\left[\frac{(1+2 \alpha)+2 \phi(1-\alpha)}{(1+2 \alpha)-\phi(1-\alpha)}\right]
$$

For the microconvection model the considered values for the calculation are,

The calculated Reynolds number $(R e)=0.0367$

Prandtl number for toluene $(P r)=7\left(@ 25^{\circ} \mathrm{C}\right)$ 


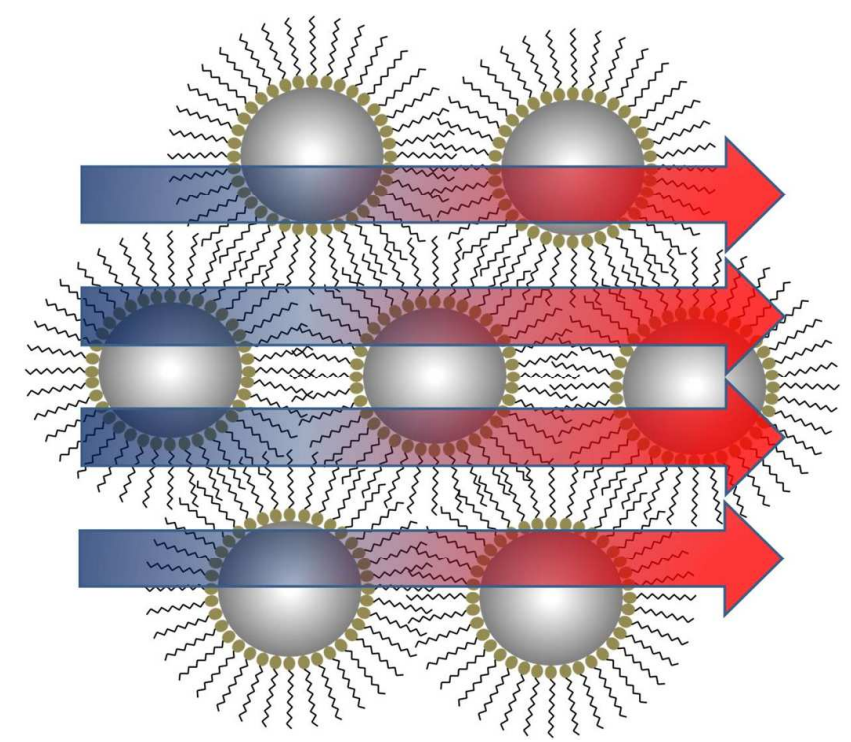

Figure S7. Schematic representation of heat transfer through clusters formed by interdigitation of the surfactant molecules attached to neighbouring nanoparticles. The direction of the arrows shown indicates the heat flow direction, the red colour indicates hot end and blue colour indicates cold end.

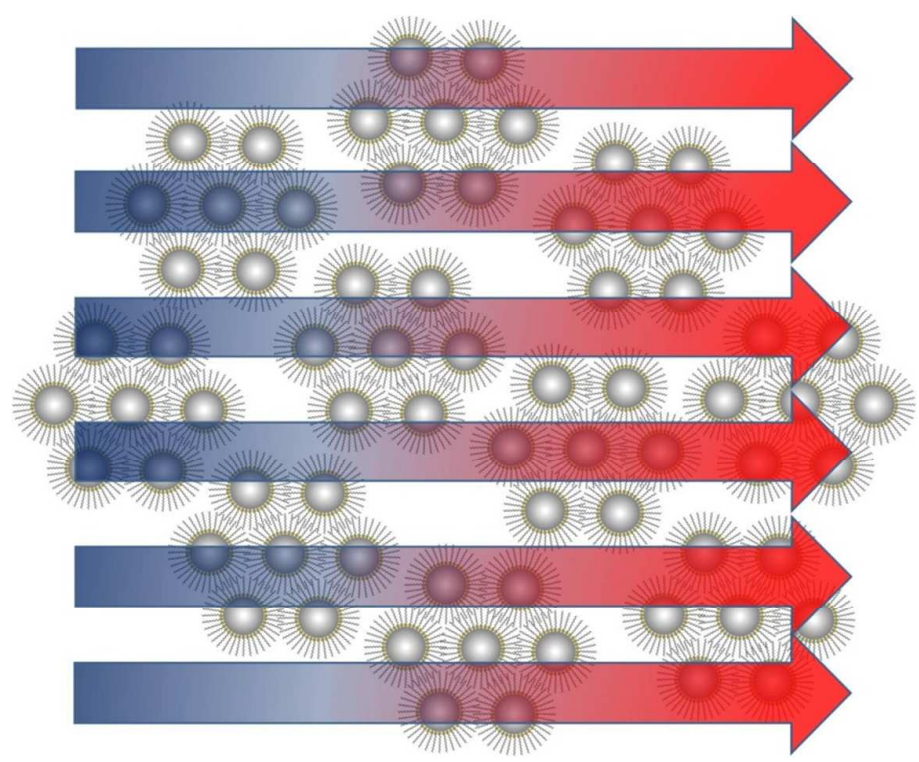

Figure S8. Schematic representation of the heat transfer through the clusters, at higher concentration of the nanoparticles in the fluid. The arrows indicate the direction of heat flow, the red colour indicates hot end and blue colour indicates cold end. 


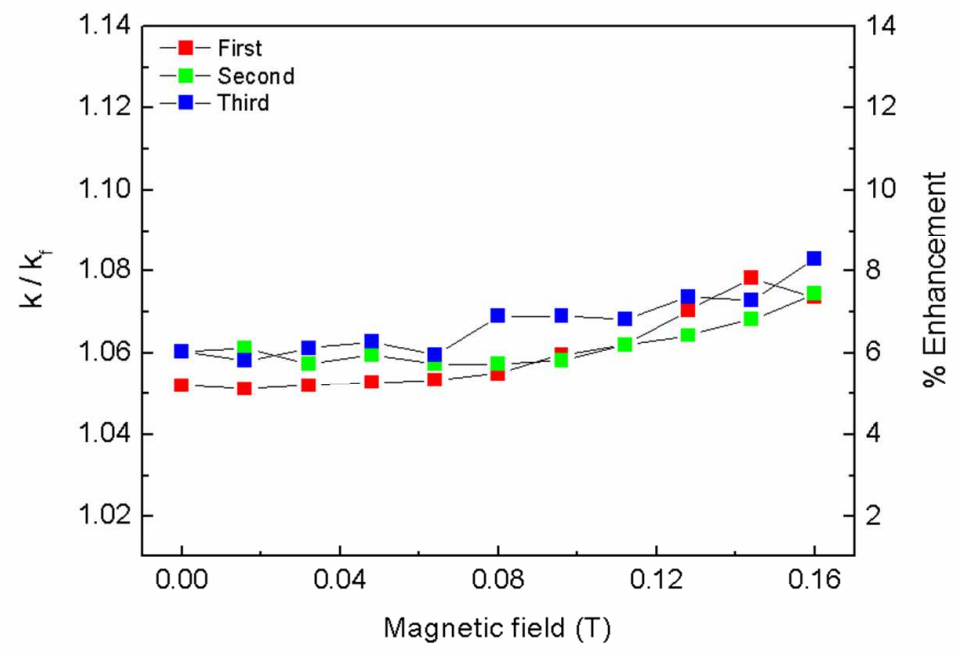

Figure S9. Repeated measurements of thermal conductivity of the less dispersible nanofluid ML2 as a function of applied magnetic field.

\section{References}

1. Lenin, R.; Joy, P. A. Effect of Particle Size Distribution on the Thermal Conductivity of Decanoic Acid Stabilized Magnetite Nanofluids. J. Nanofluids 2015, 4, 310-317.

2. Jayaprabha, K. N.; Joy, P. A. Citrate Modified $\beta$-Cyclodextrin Functionalized Magnetite Nanoparticles: A Biocompatible Platform for Hydrophobic Drug Delivery. RSC $A d v$. 2015, 5, 22117-22125.

3. Eapen, J.; Rusconi, R.; Piazza, R.; Yip, S. The Classical Nature of Thermal Conduction in Nanofluids. J. Heat Transfer 2010, 132, 102402.

4. Kauffman, G. W.; Jurs, P. C. Prediction of Surface Tension, Viscosity, and Thermal Conductivity for Common Organic Solvents Using Quantitative Structure-Property Relationships. J. Chem. Inf. Comput. Sci. 2001, 41, 408-418.

5. Ong, W.-L.; Rupich, S. M.; Talapin, D. V.; McGaughey, A. J.; Malen, J. A. Surface Chemistry Mediates Thermal Transport in Three-Dimensional Nanocrystal Arrays. Nat. Mater. 2013, 12, 410-415. 
6. Nan, C.-W.; Birringer, R.; Clarke, D. R.; Gleiter, H. Effective Thermal Conductivity of Particulate Composites with Interfacial Thermal Resistance. J. Appl. Phys. 1997, 81, 6692.

7. Minnich, A.; Chen, G. Modified Effective Medium Formulation for the Thermal Conductivity of Nanocomposites. Appl. Phys. Lett. 2007, 91, 073105.

8. Prasher, R.; Bhattacharya, P.; Phelan, P. Thermal Conductivity of Nanoscale Colloidal Solutions (Nanofluids). Phys. Rev. Lett. 2005, 94, 025901. 